\title{
Financial Sharing Framework System Based on Block Chain Technology
}

\author{
Qingquan Huang ${ }^{1, *}$ \\ ${ }^{1}$ City College of Dongguan University of Technology, Dongguan, Guangdong 523419, China \\ *Corresponding author. Email: wxxlbz@163.com
}

\begin{abstract}
In order to explore the application of blockchain technology in financial sharing, this paper takes block chain as the underlying data technology platform, by abiding by the requirements of decentralization, intellectualization, intensification and adaptability, we propose a five-level financial sharing framework system encompassing business activities, data collection, consensus contract, decentralized network architecture and sharing center, and then discuss the realization and optimization of key technologies, such as the dynamic verification of consensus mechanism, the intelligent integration of normalization rules and the expansion of efficacy, within the said framework system. Based on block chain technology, the basic procedure and information transmission model of centralized business processing of financial sharing, sustained dynamic audit and XBRL report, etc. are explored.
\end{abstract}

Keywords: block chain; financial sharing; credibility; decentralization

\section{INTRODUCTION}

At a meeting held by the Political Bureau of the Central Committee of the Communist Party of China (CPC) in October 2019, General Secretary Xi Jinping stressed that we should give full play to the important role of block chain integration technology in technological innovation and industrial reform, take block chain as an important breakthrough point for independent innovation of core technologies, clarify the main direction, increase investments and intensify efforts to crack several key technologies, and accelerate the innovative development of block chain technology and industry. This decision marked that the innovation and application development of block chain technology had been promoted to a national strategy. Since its inception in 2008, block chain technology has grown rapidly in the monetary and financial field, setting off a digital revolution about credibility. After going through the development of decentralization, smart contract and sharing economy, block chain technology has gradually become mature and applied in multiple fields. The core concept of decentralization aims to reduce the possibility that data information is tampered with and improve the credibility of information, by storing the same business on different network nodes, authenticating and maintaining in a unified way through distributed ledger keeping. The reliability safeguard mechanism of block chain technology for data information coincides with the credibility appeal of financial sharing for data information. The development of block chain technology boosts the development of sharing economy. Financial sharing must base itself on open and credible information. Nowadays, both block chain technology and financial sharing belong to emerging information technology industries, whose developments are relatively independent. How to effectively apply block chain technology to financial sharing system and reconstruct a financial sharing framework system based on block chain technology has become a significant topic in the theory and practical application of the construction of financial sharing system[1].

\section{THE DESIGN OF A FINANCIAL SHARING FRAMEWORK SYSTEM BASED ON BLOCK CHAIN TECHNOLOGY}

The fusion between block chain technology and financial shared services will radically alter the underlying data storage mode of financial shared services. The core applications of consensus mechanism and smart contract will trigger financial sharing business process and data information transmission paths. According to Michael Hammer's theory of business process reengineering, combined with the application scenarios of block chain technology, financial shared services framework system can be divided into five levels: business activities, data collection, consensus contract, decentralized network architecture and sharing center.

Business activities are the end applications of the sharing service system, comprising business activities and decision support activities[2]. On the one hand, the information generated by basic business activities is converted into data directly through the image system stored by individual block nodes, and then written into the primary chain of block chain after the consensus contract is verified. On the other hand, when basic business activities carry out 
business operations, they can directly retrieve business and financial information from adjacent nodes through the distributed network architecture, to offer a necessary reference for the implementation of business. Decision support activities include budget management, capital management and risk control, etc., which are directly linked to the knowledge base of the financial sharing center. While making decisions, the system invokes the knowledge model and optimization method of the knowledge base, and directly searches relevant data from the central server or adjacent nodes, to assist the decisionmaking process. In an effort to further highlight the intelligentization goal of sharing services, the information records of various transactions should reflect not only financial information, but also non-financial information, enrich and refine the data dictionary, improve the mapping relationship between information, and continue to refine the information ecosystem.

By relying on the image system, the data collection layer finishes business operations such as document splitting, document scanning and document filing. On functional structure, it mainly consists of OCR recognition, detection and warning, the acquisition, retrieval, classification, review, configuration, display and management of images, etc.[3]. While on process, it can be divided into two categories: automatic acquisition and manual acquisition. For automatic acquisition, the terminal scanning and input are done by an OCR recognition mechanism. Those that are successfully recognized are sorted, filed and stored according to preset rules. While data that fail to pass the verification are supplemented and completed through manual retrieval. For manual acquisition, image files are directly formed through the information input interface. It is noteworthy that the OCR recognition mechanism should fully integrate the XBRL taxonomy, embed the taxonomy, technical specifications and instance documents, and expand the taxonomy as appropriate, combined with the data element attributes of financial statement. The expansion of taxonomy requires accomplishing basic steps, such as grouping, re-establishing application scenarios, determining the logical relationships between departmental business data, teasing out national standards and tagging data attributes. In addition, this layer also needs to perform whole-process data control, such as necessary data splitting, data comparison and log management, in line with the logical relationship between data attributes and data, which lays a foundation for the application of block chain technology and the realization of financial intelligentization.

Consensus contracts are the core technology layer of information verification in financial shared services system, including two integral parts: consensus mechanism and smart contract. The consensus mechanism is a crucial channel for mutual communication and verification between various block nodes. All data originating from the image system shall be recorded in independent block nodes, and their authenticity and integrity should be verified through the consensus algorithm. Smart contracts will be triggered when code rules are met. The system mandatorily writes the data on this block node into the primary chain of block chain according to particular rules. Considering the time-sensitivity and time delay, etc. of information processing, the current consensus algorithm carries out code-based rule tests mainly through most of the adjacent nodes, rather than all nodes of the whole distributed system. The number of block chain node information feature verifications is positively correlated with the number of hash distributed nodes. Under the law of large numbers, when the nodes participating in in system operations are densely distributed, the reliability of financial shared services data can be reasonably guaranteed and basically the phenomenon of financial fraud can be eliminated. What's more, the block chain node information has specific time stamps and is arranged in a chained fashion in this order, which renders high traceability to financial information. It is worth mentioning that at present, consensus and contract technologies are still in the stage of innovation and perfection. In the construction of financial shared services center, a corresponding consensus algorithm should be selected prudently and refined constantly[4].

The sharing center is the core application layer of the sharing system. Integration and intelligent processing are carried out on traditional financial work according to the requirements of centralization and intensification, by making the most of information techniques. Sharing services are principally centralized reimbursement, centralized payment, centralized accounting, kanban and other core contents. The information processing of sharing center carries out centralized and automatic data processing on identified business, by taking basic accounting principles, XBRL taxonomy and decision information demand, etc. as the basic rules. During the construction of a decentralized distributed database, the central server is still an indispensable and integral part, which undertakes critical functions, such as the storage of core data, the recording of features of the primary chain of block chain, and real-time provision of data for the decision application layer[5]. On the design of the sharing center, we should detail and decompose the financial operation mode and operation flow, with process matrix and other tools and achieve the three-dimensional integration of business, post and personnel using information techniques. What's more, the core financial sharing platform should synergize with other business systems. It would be more complicated for conglomerates with high degree of informatization to control the specific execution process of business. All execution processes of business can be put in the ERP subsystem of department and presented in the form of kanban, so as to avoid the decentralization of data.

\section{DATA TRANSMISSION MODEL}

The introduction of block chain technology divides the architecture of the centralized financial shared services center into a binary architecture comprising a distributed node layout and a central server. They are connected to the 
engine system through consensus contracts. There are mainly three data information transmission paths: the transmission of basic business activity information, the transmission of decision support activity information and the transmission of outreach subsystem information.

Basic business activities are initiated by application terminals. A variety of operations are performed within the scope of authority and basic data are formed. The basic data are converted in the image system of the data collection layer and recorded preliminarily on the distributed server. When the block node data needs to be written into the primary chain, the system verifies them using the consensus mechanism and those that meet the conditions trigger the engine system mandatorily through smart contracts. The engine system identifies the process based on data attribute tags, and performs operations such as accounting item extraction, entry compilation, normalization of data, etc., by invoking normalization rules on the central server and eventually forms standardized business data information. Decision support activities can directly query the business data of the distributed system and central server. The distributed database only provides the function of view query of decision-related information. The specific operation and application of decision data are carried out through the engine system of the sharing center, to ensure the credibility of decision data. Outreach interfaces, such as bank-enterprise direct link system and golden tax system, are on the one hand, directly linked to the distributed system to carry out routine business operations, and on the other hand, linked to the internal business subsystems, through which they directly access the central server to finish outward data transmission, such as tax declaration and current account payments.

The binary database architecture heightens the fault tolerance risk of the system, while improving the credibility and traceability of data. The review mechanism still occupies a very important position in the system. The review scope of the review mechanism covers all kinds of block nodes, engine system, rules and standards, etc. If the fiscal and taxation policies and information taxonomy change, engines such as data classification, process standard and business extraction rule can be re-planned and modified using the review mechanism, to guarantee the timeliness of intelligent processing of financial information. In addition, under the development trend of accounting industry, i.e., business-finance integration, the financial sharing center must be integrated with other business subsystems, to allow subsystems to directly operate on the distributed system within the scope of authority and comply with the common consensus algorithm and contract, to ensure the completeness of block chain.

\section{AN ANALYSIS OF THE APPLICATION SCENARIOS OF FINANCIAL SHARING BASED ON BLOCK CHAIN TECHNOLOGY}

Before you begin to format your paper, first write and save the content as a separate text file. Keep your text and graphic files separate until after the text has been formatted and styled. Do not use hard tabs, and limit use of hard returns to only one return at the end of a paragraph. Do not add any kind of pagination anywhere in the paper. Do not number text heads-the template will do that for you.

Finally, complete content and organizational editing before formatting. Please take note of the following items when proofreading spelling and grammar:

\subsection{Centralized Business Processing}

Centralization is a core design concept in the financial sharing system. The main application scenarios include centralized reimbursement, centralized payment and centralized accounting, etc. The introduction of block chain technology reinforces the reliability and traceability of shared financial information. The consensus algorithm and smart contract become serial interfaces of the binary database architecture. Business activities are initiated by the client, and basic data are dimensionality-reduced, cleaned and converted, etc. preliminarily through the image system and stored in the distributed ledger. The distributed ledger verifies block nodes using the consensus algorithm. Once the data pass the consistency check, the smart contract will trigger the operation of various subsystems of the sharing center. In view of the impact of the probability errors of various consensus algorithms, the sharing center needs to add a re-check mechanism. After data pass the recheck, the system allocates corresponding business engines to them according to data attributes, tags and other features. The business engine checks the completeness and consistency of data attributes and supplements accordingly, pursuant to the present business-finance specifications, standards and instances, and carries out secondary data dimensionality reduction to form standard structured data. The sharing center gives intelligent business processing according to standardized entry extraction rules and business processing instances. Upon approval and review, the business and financial information is stored in the central server.

The business engine subsystems of the sharing center mainly include XBRL classification mechanism, entry extraction and centralized approval, etc. The intelligentization of entry extraction and classification mechanism depends on the intelligent learning algorithm. For this reason, the standardized data of the central server is directly connected to the engine system. On the one hand, the processing results of the business engine are stored. On the other hand, training samples can be provided for intelligent learning. The review mechanism of the sharing center is divided into two stages: access verification review 
and business result review. Access verification review is intended to ensure the reliability of the primary chain of block chain. Business result review is an important step for various functional departments to authorize approval and performance of duties, and also an important assurance for the accuracy and compliance of results. Moreover, to strengthen the information demands of different users, the client directly performs business operations on the distributed database. However, such kinds of operations are limited to the entry of original documents and application for business. Businesses like accounting, reimbursement and payment are handled by the sharing center in a centralized way. During decision-making and budgeting, the client can directly retrieve relevant data from the central server within the scope of authority, to enhance the efficiency and effect of decision support.

\subsection{Equations}

In the distributed data storage mode, independent block nodes store detailed information of each transaction. When an independent block node accesses the primary chain, it must undergo strict consensus verification, which greatly improves the reliability and traceability of information and also offers good technical support to the sustained dynamic audit of financial sharing system.

As to financial sharing audit projects, the sharing center directly retrieves data from the central server, makes compliance test and substantive test respectively, labels and records abnormal indicators. The sources of anomaly records also include fluctuations in abnormal indicators of warning system. Information and services labeled as anomaly records are locked. The information attributes are identified in accordance with taxonomy, and relevant attributes, such as keywords and tag values, are extracted. Relying on basic standard libraries such as concept sets and instance documents stored by the central server, we can carry out clustering recognition, factor analysis and other algorithms with the tag value of data as keyword, and effectively identify related business points. The transaction information in distributed ledgers is traced to the source item by item, according to the block chain value of each node, to figure out the cause of anomaly records and make appropriate audit adjustments. Finally, the sharing center automatically extracts audit information fields according to XBRL report instances and rules and fuses into an independent audit report.

In order to realize the sustained dynamic audit of financial sharing, two key problems need to be solved. One is to establish a sound warning mechanism. The centralized sharing mode has dramatically increased the business volume of the sharing center. A robust budget management system and a risk warning system are the basis to increase the standardization of auditing. The establishment of a risk warning mechanism for the sharing center should be based on budget management, highlight the interconnection between business and finance, and set a variety of risk warning indicators in an all-round, scientific and rational way, plan uniformly and implement a warning mechanism to realize real-time and sustained internal control. The other is to promote the accuracy of tracing technique of anomaly records. Block chain technology offers a reliable guarantee for the traceability of node transactions, but the association between "anomaly records-business nodes" relies on the intelligent algorithm of sharing center. Clustering analysis, multivariate neural network and other algorithms all have their specific applicable conditions. For this reason, in order to enhance the accuracy of tracing of anomaly records, we should handle basic classification and filing of information, clarify and enrich the articulation between information attributes and blend multiple intelligent traceability algorithms.

\subsection{XBRL Report}

XBRL is a standard business report metadata system comprising three basic items: concept set, taxonomy specification and instance document. In the binary database architecture, apart from the consensus contract verification mechanism between the distributed database and central server, data definition and conversion should be added as a basic section. This section takes the data after consensus contract consistency check as the basis, conducts dimensionality reduction, cleaning, keyword setting, tag assignment, etc. on the data according to the nature of business, in combination with certain taxonomy. For business and financial data, apart from specific concept value in the concept set, the relationship value of concept data is also the key to XBRL. The relationship value, on the one hand, clarifies the logical relationship between data of financial statements, and on the other hand, offers path labeling for the tracking and traceability of data. The relationship databases include resource relationship and logical relationship. The internal resource relationship is mainly connected through simple mapping between documents, while external resource relationship needs to take advantage of certain extended links. Under hierarchical classification technique, multi-point mapping logic is achieved by inheriting attributes. Logical relationships are expressed logically through concept locator, relation radian pointer and role attributes, etc., and form an interlocking relationship system.

Classification technique is the key to the implementation effect of XBRL subsystem under the financial sharing system. Classification technique needs to solve two basic problems: data element classification model algorithm and taxonomy specification. On the data element classification model algorithm, problems, such as fuzzy semantic definition, asymmetric nonlinear intelligent association and transparent modeling process, exist. Different technical standards like RDF, XML and UML will produce different data structure elements. The construction process of the overall classification algorithm pattern of XBRL should define a unified structural framework, build a two-way transmission mechanism of information classification and circulation within the unified framework system, and render a unified standard interface to the outreach system. 
On the normative system, the Ministry of Finance issued General XBRL Taxonomy in 2010 and 2015 in succession and clarified XBRL hierarchy and structured classification mode. Nevertheless, there are still some defects, such as low scalability and incomplete relational mapping. With the revision and implementation of the 2018 edition of Accounting Standards for Business Enterprises, we should draw lessons from the tracking and traceability mechanism of block chain without losing universality, further improve and clarify the XBLR taxonomy system, and keep refining the application scenario and scope of XBRL intelligence.

\section{CONCLUSION}

This project only provides a framework system for the integration between block chain technology and financial sharing system, and discusses the application scenarios in terms of process and mode. Nevertheless, considering many uncertainties in the development trend and consensus standard of block chain technology, specific details about the construction of consensus contract algorithm, attribute expansion of XBRL concept set, and interface integration mechanism with the outreach system have not been touched upon. The applications of block chain technology in finance, credit investigation and other fields have yielded fruitful results. With further development of block chain and financial sharing technology, policies, regulations and standards should be further refined. The construction of the financial sharing system based on block chain technology will continue to go deep into bottom layer. Breakthroughs are bound to be made in data storage, relationship building, consensus algorithm and intelligent information extraction, etc., which is also an important direction for the follow-up research on this topic.

\section{ACKNOWLEDGMENT}

Funded Programs: Innovative Young Talent Program in Regular Institutions of Higher Learning in Guangdong Province, Research on the Innovative Development of Blockchain Industry in the Guangdong-Hong Kong-Macao Greater Bay Area, Program No.: 2019WQNCX153;

Fund Program for the Development of Young Teachers in City College of Dongguan University of Technology in 2019, On Risk Management of P2P Online Lending Platform Based on COSO-ERM Framework, Program No.: 2019QJY011R.

\section{REFERENCES}

[1] Yang Runhui. Application of Block Chain Technology in the Field of Financial Sharing [J]. Finance and Accounting Monthly, 2020 (09): 35-40.

[2] Geng Yucheng. On Optimization Strategies of Management Accounting Information System from the Perspective of Financial Sharing [J]. Chinese Enterprise Accounting of Villages and Towns, 2019 (01): 248-251.

[3] Li Kehong. On the Framework of Smart Financial Management Mode from the Perspective of Artificial Intelligence [J]. Friends of Accounting, 2020 (05): 5962.

[4] Wang Xiuping, Zhang Tianyi. Defects in Financial Shared Services and Countermeasures [J]. Finance and Accounting Monthly, 2012 (20): 70-71.

[5] Hao Wentao. On Distributed Fast Trading Framework Based on Block Chain [D], Lanzhou University of Technology. 2019. 